\title{
Pelvic angiography is effective for emergency pediatric patients with pelvic fractures: a propensity-score-matching study with a nationwide trauma registry in Japan
}

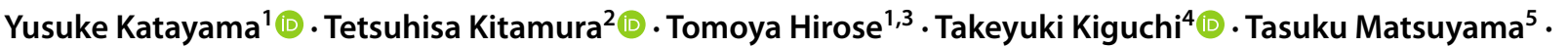 \\ Hiroki Takahashi ${ }^{6} \cdot$ Kosuke Kiyohara $^{7}$ (1) Junya Sado ${ }^{8} \cdot$ Shingo Adachi $^{9} \cdot$ Tomohiro Noda $^{10} \cdot$ Junichi Izawa ${ }^{11}$ (i) \\ Yuko Nakagawa ${ }^{1} \cdot$ Takeshi Shimazu $^{1}$
}

Received: 6 December 2018 / Accepted: 15 May 2019 / Published online: 22 May 2019

(c) Springer-Verlag GmbH Germany, part of Springer Nature 2019

\begin{abstract}
Purpose The aim of this study was to evaluate the association between the implementation of pelvic angiography (PA) and outcome in emergency pediatric patients with pelvic fracture.

Methods We extracted data on pelvic fracture patients aged $\leq 19$ years between 2004 and 2015 from a nationwide trauma registry in Japan. The main outcome was hospital mortality. We assessed the relationship between implementation of PA and hospital mortality using one-to-one propensity-score-matching analysis to reduce potential confounding effects in comparing the PA group with the non-PA group.

Results In total, 1351 patients were eligible for our analysis, with 221 patients (16.4\%) included in the PA group and 1130 patients (83.6\%) included in the non-PA group. For all patients, the proportion of hospital mortality was higher in the PA group than in the non-PA group [13.6\% (30/221) vs 7.1\% (80/1130), crude odds ratio (OR) 2.062 (95\% confidence interval (CI), 1.318-3.224); $p=0.002]$. In the propensity-score-matched patients, the proportion of hospital mortality was lower in the PA group than in the non-PA group [10.5\% (22/200) vs 18.2\% (38/200), $p=0.027]$. This finding was confirmed in both the multivariable logistic regression model [adjusted OR 0.392 (95\% CI, $0.171-0.896$ ); $p=0.026$ ] and the conditional logistic regression model [conditional OR 0.484 (95\% CI, 0.261-0.896); $p=0.021$ ].

Conclusion The implementation of PA was significantly associated with lower hospital mortality among emergency pediatric patients with pelvic fractures compared with the non-implementation of PA.
\end{abstract}

Keywords Pelvic fracture $\cdot$ Angiography $\cdot$ Children $\cdot$ Shock $\cdot$ Propensity-score matching

Yusuke Katayama

orion13@hp-emerg.med.osaka-u.ac.jp

1 Department of Traumatology and Acute Critical Medicine, Osaka University Graduate School of Medicine, 2-15

Yamada-oka, Suita 565-0871, Japan

2 Division of Environmental Medicine and Population Sciences, Department of Social and Environmental Medicine, Osaka University Graduate School of Medicine, 2-15 Yamada-oka, Suita, Japan

3 Emergency and Critical Care Center, Osaka Police Hospital, 10-31 Kitayama-cho, Tennoji-ku, Osaka, Japan

4 Kyoto University Health Services, Yoshida-honmachi, Sakyo-ku, Kyoto, Japan

5 Department of Emergency Medicine, Kyoto Prefectural University of Medicine, 465 Kajiicho, Hiroko-ji noboru, Kawaramachi-dori, Kamigyo-ku, Kyoto, Japan
6 Department of Emergency and Critical Medicine, Kansai Medical University, 2-3-1 Shin-machi, Hirakata, Japan

7 Department of Food Science, Faculty of Home Economics, Otsuma Women's University, 12 Sanban-cho, Chiyoda-ku, Tokyo, Japan

8 Department of Health and Sport Sciences, Medicine for Sports and Performing Arts, Osaka University Graduate School of Medicine, 2-15 Yamada-oka, Suita, Japan

9 Rinku General Medical Center, Senshu Trauma and Critical Care Center, 2-23 Rinku Orai-kita, Izumisano, Japan

10 Department of Traumatology and Critical Care Medicine, Osaka City University Graduate School of Medicine, 1-5-7 Asahi-machi, Abeno-ku, Osaka, Japan

11 Intensive Care Unit, Department of Anesthesiology, The Jikei University School of Medicine, 3-19-18 Nishi-Shinbashi, Minato-ku, Tokyo, Japan 


\section{Introduction}

Pelvic fracture is one of the severe traumas associated with high mortality due to fatal bleeding. There are several treatment strategies such as external fixation, retroperitoneal pelvic packing (RPP), and transcatheter arterial embolization (TAE) plus pelvic angiography (PA). According to the Eastern Association for the Surgery of Trauma (EAST) guidelines, emergency PA is recommended for adult patients with pelvic fractures who show hemodynamic instability, signs of ongoing bleeding after non-pelvic sources of blood loss, or arterial intravenous contrast extravasation in the pelvis by enhanced computed tomography (CT) [1]. Indeed, Falzarano and colleagues reported that $16.5 \%(87 / 528)$ of pelvic fracture patients had hemodynamic instability, and 11.9\% (63/528) of them received emergency angiography and embolization [2].

However, pelvic fractures are rarer among children than among adults; the incidence is between $2.4 \%$ and $7.5 \%$ [3-5]. It is difficult to select appropriate sheathes and catheters for pediatric patients with pelvic fractures, because the body size of children is smaller than that of adults. Therefore, angiography or arterial embolization for emergency pediatric patients with pelvic fractures is rarely performed. Although there were some case series and case reports on angiography and catheter embolization in these patients [6-10], the effectiveness of PA in them has not been extensively investigated.

The Japanese Trauma Data Bank (JTDB) is a nationwide hospital-based trauma registry in Japan [11]. Using this database, we evaluated the association between the implementation of PA and outcome among emergency pediatric patients with pelvic fractures, based on a propensity-score (PS)-matching method.

\section{Methods}

\section{Study design, population, and setting}

This study was a retrospective observational study using the JTDB database. The study period spanned 12 years from January 2004 to December 2015. We included emergency pediatric patients aged $\leq 19$ years who had pelvic fractures, were then transported to JTDB-participating hospitals, and were registered in the database. We extracted pediatric patients with pelvic fractures based on the following Abbreviated Injury Scale (AIS) codes: $852,600.2,852,602.2,852,604.3,852,606.4,852,608.4$, $852,610.5,852,800.3$, and $853,000.3$. In addition, we excluded those who were in cardiopulmonary arrest on hospital arrival, underwent inter-hospital transport, and had missing information on factors such as vital signs on hospital arrival, sex, outcome, or Injury Severity Score (ISS). This study defined patients in cardiopulmonary arrest as those whose systolic blood pressure was $0 \mathrm{mmHg}$ and/or heart rate was $0 \mathrm{bpm}$ on hospital arrival. This study was approved by the ethics committee of Osaka University Graduate School of Medicine. Personal identifiers were removed beforehand from the JTDB database, and thus, the patients' right to informed consent was waived. This manuscript was written based on the STROBE statement to assess the reporting of cohort and cross-sectional studies [12].

\section{Japanese trauma data bank}

The JTDB was established by the Japanese Association for the Surgery and Trauma (Trauma Surgery Committee) and the Japanese Association for Acute Medicine (Committee for Clinical Care Evaluation) $[13,14]$ and is similar to trauma databases in North America, Europe, and Oceania [15]. By 2016, 256 major emergency medical institutions around Japan had been registered in the JTDB database [14]. These hospitals have an ability equal to Level I trauma centers in the United States. Data were collected via the Internet from participating institutions. In most cases, the physicians and technicians who attended an AIS coding course had registered the data [15].

The JTDB captures data on trauma patients that includes age, sex, mechanism of injury, AIS code (version 1998), ISS, vital signs on hospital arrival, date and some time series from hospital arrival to discharge, and medical treatments such as interventional radiology and CT scanning and complications in accordance with regular forms for coding items [16]. The ISS was calculated from the top three scores of the AIS for nine sites classified by the AIS code.

From the JTDB database, we extracted age, sex, time of day and day of the week of hospital admission, calendar year, shock on hospital arrival, mechanism of injury, ISS, existence of injury with AIS score of three or more for each site, and implementation of PA. This study defined daytime as 09:00 am to 17:59 pm and nighttime as 18:00 pm to 08:59 am and also defined shock as a systolic blood pressure below $80 \mathrm{mmHg}$ on hospital arrival [17].

\section{Endpoint}

The main endpoint was hospital mortality.

\section{Statistical analysis}

Pre- and in-hospital information and outcome were evaluated between the PA group and the non-PA group. To reduce 
potential confounding effects in the comparisons between the two groups, we estimated a PS by fitting a logistic regression model that was adjusted for the following nine variables before the decision to conduct PA was made: age (continuous variable), sex (male or female), time of day (daytime, nighttime, unknown), day of the week (weekday or weekend), shock on hospital arrival (presence or absence), ISS (continuous variable), existence of injury with AIS score of three or more for each site (yes or no), mechanism of injury (car occupant, motorcycle, bicycle, pedestrian, fall from high place, other, and unknown), and calendar year (2004-2006, 2007-2009, 2010-2012, and 2013-2015). We performed a receiver operating characteristic curve analysis with an area under the curve of PS for predicting the implementation of PA among the patients with pelvic fractures. One-to-one pair matching between the PA group and the non-PA group was performed by nearest-neighbor matching without replacement, using calipers of width equal to 0.2 of the standard deviation mean differences (SMD) before and after matching. When the SMD was less than 0.25 [18], we considered this to indicate a negligible imbalance between the two groups.

We investigated the association between the implementation of PA and hospital mortality among pediatric patients with pelvic fractures using univariable, multivariable, and conditional logistic regression analyses. On the basis of these analyses, we calculated their odds ratios (ORs) with 95\% confidential intervals (CIs). In the multivariable logistic regression model, we adjusted for the above nine variables used in the PS calculation. In addition, we performed a subgroup analysis by shock status on hospital arrival (presence or absence). All tests were two-tailed, and $p$ values of $<0.05$ were considered statistically significant. All statistical analyses were performed with the use of SPSS version 23.0 J (IBM Corp., Armonk, NY) and R version 3.1.0 (The R Foundation for Statistical Computing).

\section{Results}

Figure 1 shows the patient flow in this study. During the study period, 226,698 emergency patients were registered in the JTDB database, and 24,485 patients suffered pelvic fractures. Of them, 22,793 patients were $\geq 20$ years, and 1692 patients were $\leq 19$ years. After excluding 341 patients for the reasons shown in Fig. 1, 1351 patients were eligible for our analysis, with 221 patients (16.4\%) included in the PA group and 1130 patients $(83.6 \%)$ included in the non-PA group.

The clinical characteristics of the eligible patients in the PA group and the non-PA group before and after PS matching are shown in Table 1. Among all patients before PS matching, those in the PA group were more likely to be older, have shock on hospital arrival and a high ISS score, and to have received injuries to the abdomen and lower extremities including the pelvis with an AIS score of three or more compared with those in the non-PA group. As for the mechanism of injury, the proportion of patients with a fall from a high place was higher, but that of car occupant, bicycle, and pedestrian were lower in the PA group than in the non-PA group. After PS matching, 200 patients from each group were selected, and the area under the receiver operating characteristic curve of the PS was 0.823. The covariate balance between the two groups of matched patients improved greatly.

Table 2 shows the relationship between the implementation of PA and hospital mortality among the
Fig. 1 Flowchart of patient enrollment

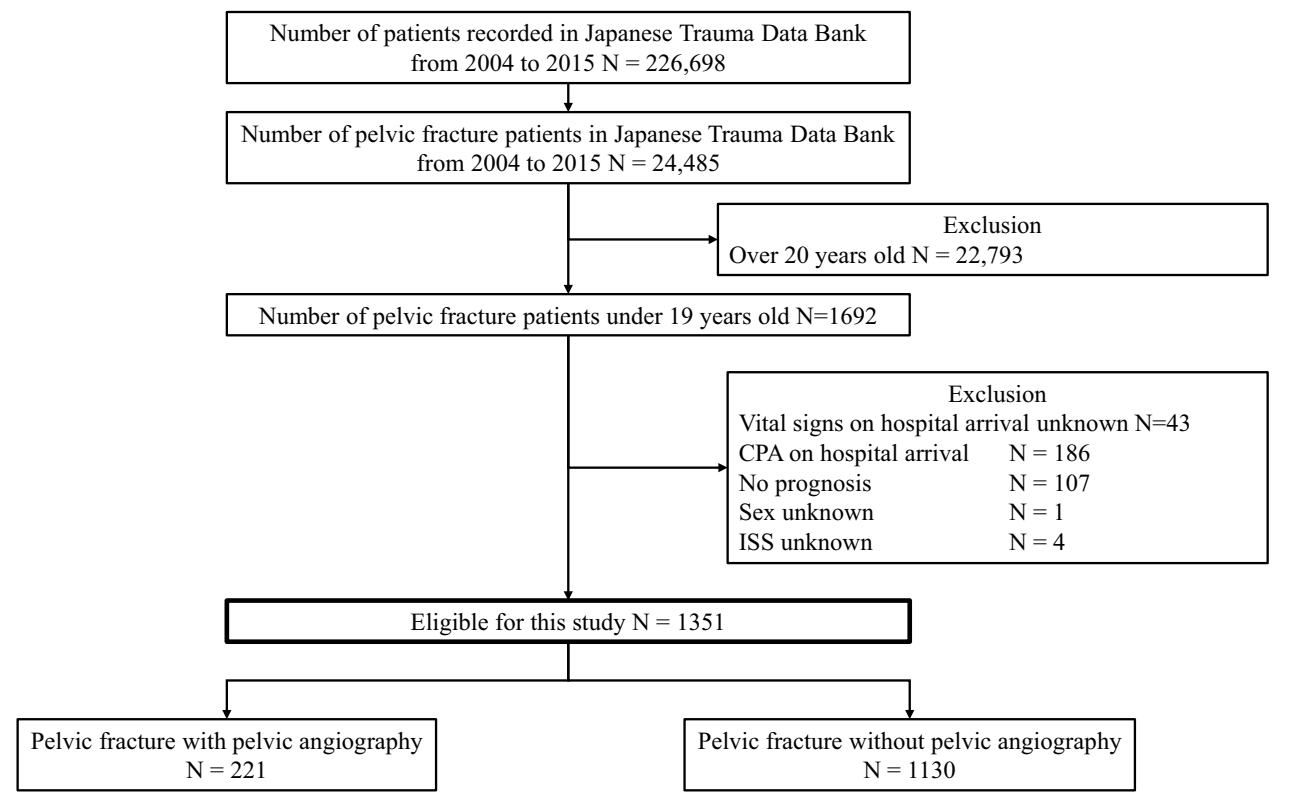


Table 1 Patient characteristics among all cohorts and propensity-score-matched cohort

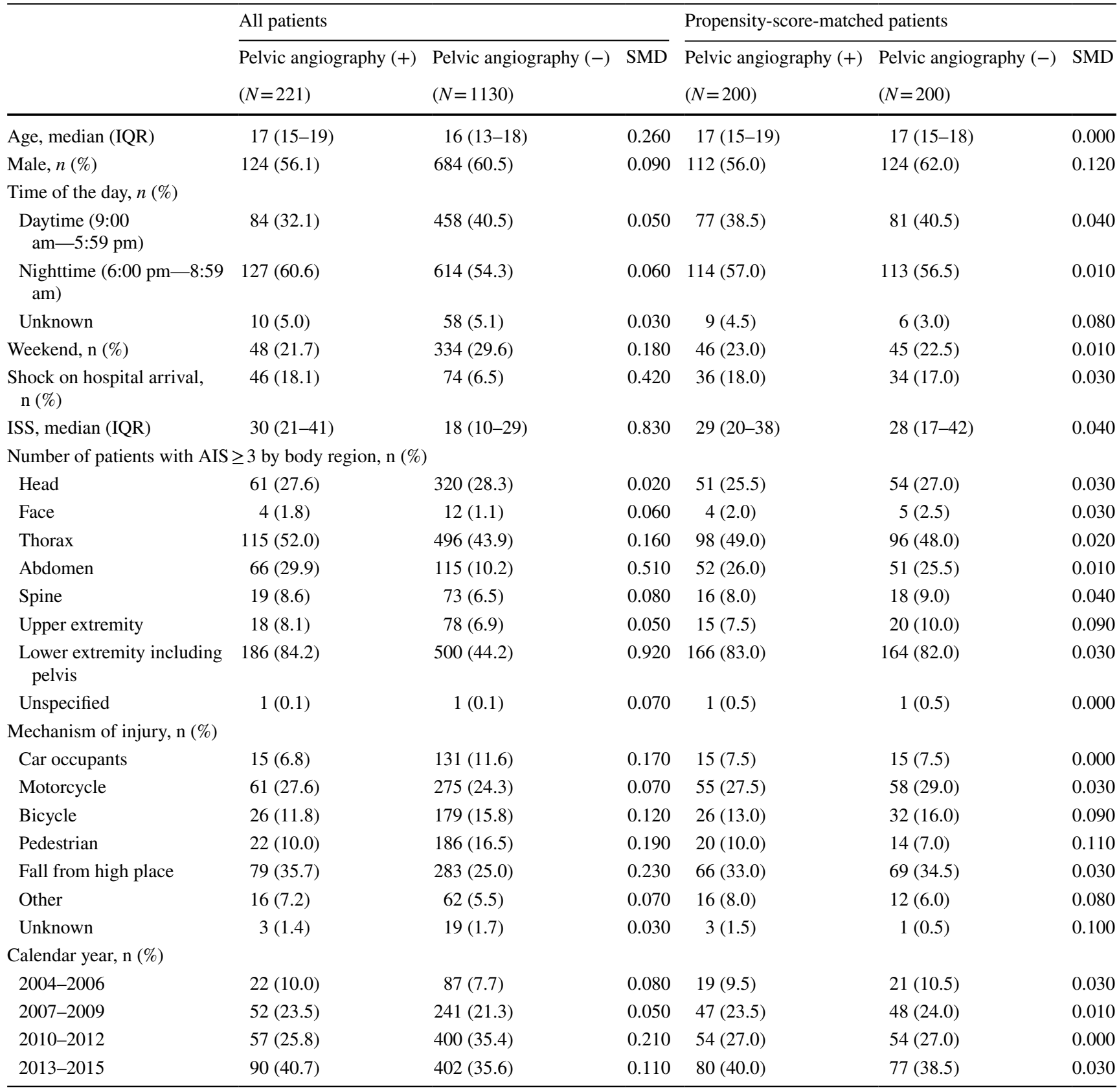

The area under the receiver operating characteristic curve of the logistic regression model to calculate a propensity score was 0.823

$I Q R$ interquartile range, ISS injury severity score, $A I S$ abbreviated injury scale, SMD standard mean difference

pediatric emergency patients with pelvic fractures. Among all patients before PS matching, the proportion of hospital mortality was higher in the patients in the PA group than in those in the non-PA group [13.6\% $(30 / 221)$ vs $7.1 \%(80 / 1130)$, crude OR 2.062 (95\% CI $1.318-3.224) ; p=0.002]$. However, the PA group tended to have a low OR for hospital mortality in the multivariable logistic regression model [adjusted OR 0.684 (95\% CI 0.346-1.354); $p=0.276]$. In the PS matched patients, hospital mortality was lower in the PA group than in the non-PA group [10.5\% (22/200) vs $18.2 \%(38 / 200)$, crude OR 0.527 (95\% CI 0.299-0.929); $p=0.027$ ], and there were statistically significant differences between the PA group and the non-PA group in both the multivariable logistic regression model [adjusted OR 0.392 (95\% CI $0.171-0.896) ; p=0.026]$ and the conditional logistic regression model [conditional OR 0.484 (95\% CI $0.261-0.896) ; p=0.021]$. 
Hospital mortality between the two groups of the pediatric patients with pelvic fractures by shock status on hospital arrival is shown in Table 3. Among the patients without shock on hospital arrival, hospital mortality was higher in the PA group than in the non-PA group [9.2\% (16/174) vs 4.7\% (49/1049), crude OR 2.067 (95\% CI 1.147-3.724)]. However, among the patients with shock on hospital arrival, $\mathrm{t}$ hospital mortality was lower in the PA group than in the non-PA group [30.4\% (14/46) vs 39.2\% (29/74), OR 0.679 (95\% CI 0.310-1.485)], and the $p$ for interaction was 0.026 .

\section{Discussion}

From the data of a nationwide trauma registry in Japan, this study showed that the implementation of PA was significantly associated with lower hospital mortality among emergency pediatric patients aged $\leq 19$ years with pelvic fractures compared with non-implementation of PA. This result from a matched cohort after PS matching was almost similar to that from the entire patient cohort before PS matching, which reinforced the robustness of our results. This study showing the effectiveness of the angiographic assessment of pediatric patients with pelvic fractures provides important clues for discussing treatment strategies in these patients.

This study revealed that the implementation of PA was associated with a low proportion of hospital mortality among emergency pediatric patients with pelvic fractures. As the ability of CT scanning has continued to advance, its role has become important in the initial assessment of emergency trauma patients. Especially, extravasation confirmed by enhanced CT scanning is effective for detecting arterial bleeding, and PA is recommended for pelvic fracture patients with extravasation confirmed by enhanced CT scanning in the EAST guidelines [1]. However, some studies reported that emergency patients with abdominal traumas and/or pelvic fractures could be conservatively managed if their conditions were stable, even if there was extravasation on enhanced CT scanning [19-21]. Conversely, in another report, even if no extravasation was apparent on enhanced CT scanning, TAE for arterial bleeding confirmed by angiography was conducted and subsequently led to an improvement of patient outcome [21]. Thus, making a decision about treatment strategies such as embolization and surgery based solely on enhanced CT scanning might not be appropriate emergency care. Although we did not obtain information on extravasation on enhanced CT scanning, the present study suggested that the evaluation of arterial bleeding with the use of angiography in pediatric patients with pelvic fractures was effective regardless of the findings on enhanced CT scanning.

As with adult patients with pelvic fractures, some studies reported that external fixation and both RPP and TAE were 
Table 3 Subgroup analysis of death at hospital discharge among pediatric pelvic fractures patients with or without pelvic angiography

\begin{tabular}{|c|c|c|c|c|c|c|c|}
\hline & \multicolumn{2}{|c|}{$\begin{array}{l}\text { Pelvic angiography } \\
(+)\end{array}$} & \multicolumn{2}{|c|}{$\begin{array}{l}\text { Pelvic angiography } \\
(-)\end{array}$} & \multirow[t]{2}{*}{ Crude OR } & \multirow[t]{2}{*}{$(95 \% \mathrm{CI})$} & \multirow[t]{2}{*}{$\begin{array}{l}P \text { Value for } \\
\text { interaction }\end{array}$} \\
\hline & $\%(n / N)$ & & $\%(n / 1$ & & & & \\
\hline $\begin{array}{l}\text { Shock on } \\
\text { hospital } \\
\text { arrival* }\end{array}$ & & & & & & & 0.026 \\
\hline Shock (-) & 9.2 & $(16 / 174)$ & 4.7 & $(49 / 1049)$ & 2.067 & $(1.147-3.724)$ & \\
\hline Shock (+) & 30.4 & $(14 / 46)$ & 39.2 & $(29 / 74)$ & 0.679 & $(0.310-1.485)$ & \\
\hline
\end{tabular}

$O R$ odds ratio, $C I$ confidence interval

*Systolic blood pressure $\leq 80 \mathrm{mmHg}$ also effective for pediatric patients [8, 9, 22, 23]. Angiography allows the detection and simultaneous treatment of arterial bleeding, and its implementation would be more effective for children. Recently, CT-guided surgery was reported for tile type C pelvic injury [24]. As the blood flow in children is less than that in adults, bloodless treatments such as CT-guided surgery and embolization might be effective for pediatric patients with pelvic fracture. Although it is difficult to select an appropriate sheath and/or catheter size for pediatric patients when angiography is performed, according to case studies of TAE performed in children with hepatic injury, splenic injury or pelvic fracture, TAE could be performed without complications such as vessel laceration or thrombosis [25-27]. Thus, if operators can select an appropriate catheter size for pediatric patients, angiography could be performed safely without complications.

In the subgroup analysis, hospital mortality among the pediatric pelvic fracture patients with shock on hospital arrival was lower in the PA group than in the non-PA group. In adult patients with pelvic fractures, some studies pointed out that various factors such as hemodynamic instability need to be considered before performing emergency angiography [28-32]. Therefore, among patients with shock on hospital arrival, the identification of arterial bleeding by proactive PA would lead to the simultaneous implementation of TAE and, subsequently, a better outcome. However, hospital mortality among those without shock on hospital arrival was higher in the PA group than in the non-PA group. Although the reasons for the implementation of PA were unclear in this study, the PA group might have had severe conditions for which arterial bleeding was suspected, and/ or the non-PA group might have had minor pelvic fractures that did not require PA.

\section{Limitations}

This study has several limitations. First, it assessed the relationship between PA and hospital mortality among pediatric patients with pelvic fractures but did not assess the reasons for the implementation of PA such as extravasation on enhanced CT scanning. Second, as there was also no information recorded on external fixation or RPP or on the type of pelvic fractures based on the AO/OTA classification [33] in the JTDB database, the influence of these factors on patient outcome was unknown. Third, it was unclear whether the cause of death was due to bleeding or other reasons such as traumatic brain injury and infection. Fourth, although nine variables were used to calculate the PS, there may be other factors that affect the implementation of PA. For example, factors such as hospital treatment protocols and decision-making by surgeons may affect the implementation of PA, but these data were not available in the JTDB database. However, the area under the receiver operating characteristic curve of PS was 0.823 in this model, which would sufficiently explain the implementation of PA. Finally, because this study was a retrospective observational study, there might be unknown confounding factors that influence the relationship between PA and hospital mortality.

\section{Conclusion}

In this patient population, the implementation of PA was significantly associated with lower hospital mortality among emergency pediatric patients with pelvic fractures compared with the non-implementation of PA.

Acknowledgements The authors thank the emergency medical service personnel, nurses and emergency physicians who participated in the JTDB. This article was supported by the Clinical Investigator's Research Project in Osaka University Graduate School of Medicine.

Data availability The data that support the findings of this study are available from the JTDB, but the availability of these data is restricted.

\section{Compliance with ethical standards}

Conflict of interests The authors declare that they have no competing of interests. 
Ethics approval and consent to participate This study was approved by the ethics committees of the Osaka Graduate School of Medicine (No. 16260), and the requirement to obtain patients' consent to participate was waived, because the data were anonymous.

Open Access This article is distributed under the terms of the Creative Commons Attribution 4.0 International License (http://creativeco mmons.org/licenses/by/4.0/), which permits unrestricted use, distribution, and reproduction in any medium, provided you give appropriate credit to the original author(s) and the source, provide a link to the Creative Commons license, and indicate if changes were made.

\section{References}

1. Cullinane DC, Schiller HJ, Zielinski MD, et al. Eastern association for the surgery of trauma practice management guidelines for hemorrhage in pelvic fracture-update and systematic review. J Trauma. 2011;71:1850-68.

2. Falzarano G, Medici A, Carta S, et al. The orthopedic damage control in pelvic ring fractures: when and why-a multicenter experience of 10 years' treatment. J Acute Dis. 2014;3:201-6.

3. Schlickewei W, Keck T. Pelvic and acetabular fractures in childhood. Injury. 2005;36(Suppl 1):A57-63.

4. Ismail N, Bellemare JF, Mollitt DL, et al. Death from pelvic fracture: children are different. J Pediatr Surg. 1996;31:82-5.

5. Demetriades D, Karaiskakis M, Velmahos GC, et al. Pelvic fractures in pediatric and adult trauma patients: are they different injuries? J Trauma. 2003;54:1146-51 discussion 1151.

6. Kiankhooy A, Sartorelli KH, Vane DW, et al. Angiographic embolization is safe and effective therapy for blunt abdominal solid organ injury in children. J Trauma. 2010;68:526-31.

7. Ohtsuka Y, Iwasaki K, Okazumi S, et al. Management of blunt hepatic injury in children: usefulness of emergency transcatheter arterial embolization. Pediatr Surg Int. 2003;19:29-34.

8. Puapong D, Brown CV, Katz M, et al. Angiography and the pediatric trauma patient: a 10 -year review. J Pediat Surg. 2006;41:1859-63.

9. Vo NJ, Althoen M, Hippe DS, et al. Pediatric abdominal and pelvic trauma: safety and efficacy of arterial embolization. J Vasc Interv Radiol. 2014;25:215-20.

10. Gonzalez KW, Dalton BG, Kerisey MC, et al. Pelvic angiography for trauma in children: a rare but useful adjunct. Eur J Pediatr Surg. 2017;27:196-9.

11. Japanese Trauma Data Bank. https://www.jtcr-jatec.org/traum abank/index.htm. Accessed 12 Sep 2018.

12. Von Elm E, Altman DG, Egger M, et al. STROBE Initiative. The strengthening the reporting of observational studies in epidemiology (STROBE) statement: guidelines for reporting observational studies. J Clin Epidemiol. 2008;61:344-9.

13. Yumoto T, Mitsuhashi T, Yamakawa Y, et al. Impact of cushing's sign in the prehospital setting on predicting the need for immediate neurosurgical intervention in trauma patients: a nationwide retrospective observational study. Scand J Trauma Resusc Emerg Med. 2016;24:147.

14. Japan Trauma Care and Research. Japan Trauma Data Bank Annual Report 2011-2015. https://www.jtcr-jatec.org/traumabank /dataroom/data/JTDB2016.pdf. Accessed 3 Sep 2018.

15. Shoko T, Shiraishi A, Kaji M, et al. Effect of pre-existing medical conditions on in-hospital mortality: analysis of 20,257 trauma patients in Japan. J Am Coll Surg. 2010;211:338-46.
16. Nakada TA, Nakao S, Mizushima Y, et al. Association between male sex and increased mortality after falls. Acad Emerg Med. 2015;222:708-13.

17. Neidel T, Salvador N, Heller RA. Impact of systolic blood pressure limits on the diagnostic value of triage algorithms. Scand J Trauma Resusc Emerg Med. 2017;25:118.

18. Stuart EA. Matching methods for causal inference: a review and a look forward. Stat Sci. 2010;25:1-21.

19. Bansal S, Karrer FM, Hansen K, et al. Contrast blush in pediatric blunt splenic trauma does not warrant the routine use of angiography and embolization. Am J Surg. 2015;210:345-50.

20. Juern JS, Milia D, Codner P, et al. Clinical significance of computed tomography contrast extravasation in blunt trauma patients with a pelvic fracture. J Trauma Acute Care Surg. 2017;81:138-40.

21. Verbeek DO, Zijlstra IA, van der Leij C, et al. Management of pelvic ring fracture patients with a pelvic "blush" on early computed tomography. J Trauma Acute Care Surg. 2014;76:374-9.

22. Burlew CC, Moore EE, Smith WR, et al. Preperitoneal pelvic packing/external fixation with secondary angioembolization: optimal care for life-threatening hemorrhage from unstable pelvic fractures. J Am Coll Surg. 2011;212:628-35 (discussion 635-7).

23. Chao NS, Liu CS, Chung KL, et al. Retroperitoneal pelvic packing for haemodynamically unstable pelvic fractures in children and adolescents: a level-one trauma-centre experience. J Pediatr Surg. 2012;47:2244-50.

24. Falzarano G, Rollo G, Bisaccia M, et al. Percutaneous screws CT guided to fix sacroiliac joint in tile $\mathrm{C}$ pelvic injury. Outcomes at 5 years of follow-up. SICOT J. 2018;4:52.

25. Qasim ZA, Masood R, Mateen MA. Successful use of angiographic embolization to control hemorrhage from blunt pelvic trauma in a pediatric patient. J Pak Med Assoc. 2004;54:32-3.

26. Gonzalez KW, Dalton BG, Kerisey MC, et al. Pelvic angiography for trauma in children: a rare but useful adjunct. Eur J Pediatr Surg. 2017;27:196-299.

27. Ong CC, Toh L, Lo RH, et al. Primary hepatic artery embolization in pediatric blunt hepatic trauma. J Pediatr Surg. 2012;47:2316-20.

28. Eastridge BJ, Starr A, Minei JP, et al. The importance of fracture pattern in guiding therapeutic decision-making in patients with hemorrhagic shock and pelvic ring disruptions. J Trauma. 2002;53:446-50 (discussion 450-1).

29. Lopez PP. Unstable pelvic fractures: the use of angiography in controlling arterial hemorrhage. J Trauma. 2007;62:S30-1.

30. Salim A, Teixeira PG, DuBose J, et al. Predictors of positive angiography in pelvic fractures: a prospective study. J Am Coll Surg. 2008;207:656-62.

31. El-Haj M, Bloom A, Mosheiff T, et al. Outcome of angiographic embolization for unstable pelvic injuries: factors predicting success. Injury. 2013;44:1750-5.

32. Lai $\mathrm{YC}, \mathrm{Wu} \mathrm{CH}, \mathrm{Chen} \mathrm{HW}$, et al. Predictors of active arterial hemorrhage on angiography in pelvic fracture patients. Jpn J Radiol. 2018;36:223-30.

33. March JL, Slongo TF, Agel J, et al. Fracture and dislocation classification compendium-2007: orthopaedic Trauma association classification, database and outcomes committee. J Orthop Trauma. 2007;21(10 Suppl):S1-133. 